\title{
One Step Synthesis of 6-Amino-5-Cyano-4-Phenyl-2-Mercapto Pyrimidine Using Phosphorus Pentoxide
}

\author{
Dipti R. Patil, Sonali M. Salunkhe, Madhukar B. Deshmukh, and Prashant V. Anbhule*
}

Department of Chemistry, Shivaji University, Kolhapur- 416 004, Maharashtra, India

\begin{abstract}
A simple and efficient approach towards one step synthesis of 6-amino-5-cyano-4-phenyl-2-mercapto pyrimidine and its hydroxyl derivatives have been developed by three component condensation of aromatic aldehydes, malononitrile and thiourea/urea in presence of phosphorus pentoxide (Scheme 1).
\end{abstract}

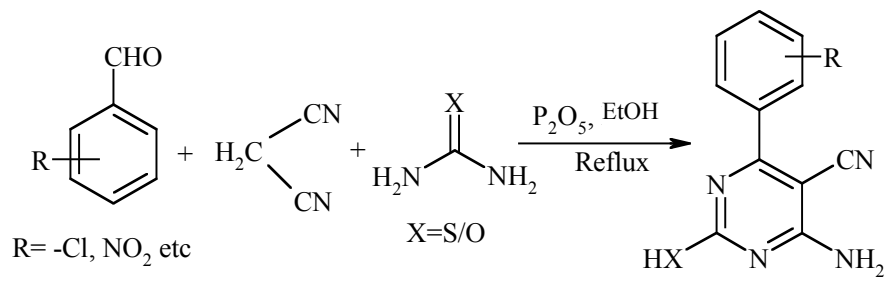

Scheme 1.

Keywords: Phosphorus pentoxide, urea/thiourea, pyrimidine one step synthesis.

\section{INTRODUCTION}

Heterocyclic molecules are of biological interest due to their potential physical and chemical properties [1]. Among these the pyrimidine compounds occupy a unique position in pharmaceutical chemistry, as they are components of nucleic acids. The important pyrimidine compounds have diverse applications as bactericidal [2], fungicidal [3], analgesics [4], anti-inflammatory [5] and anti tumor agents [6].

Nowadays, the one step methods involving three component condensation using different reagents and catalysts are popular in synthetic organic chemistry for the synthesis of heterocyclic compounds. These single step methods are more convenient as compared with two step strategies as they require shorter reaction times, product isolation easy and give higher yields and recoveries of the product.

Although a number of papers have been reported concerning the synthesis of pyrimidine derivatives [7, 8], few one pot synthesis $[9,10]$ have been published using aromatic aldehydes, cyano ethyl acetate and thiourea.

Peter Russell and George H. Hitchings prepared 2,4,6triaminopyrimidines by refluxing malononitrile with guanidine in alcohol [11]. Biginelli reported one-step synthesis of 3,4-dihydro pyrimidones by three- component condensation of aldehydes, ethyl acetoacetate and urea [12] in alcoholic medium using strong mineral acid. These compounds possess several pharmaceutical properties like antibacterial, antiviral, anti-inflammatory, anti-hypertensive

*Address correspondence to this author at the Department of Chemistry, Shivaji University, Kolhapur- 416 004, Maharashtra, India; Fax: +91-02312692333; E-mail: pvanbhule@gmail.com and anti- tumor agents $[13,14]$. They also serve as calcium channel blockers, as $\alpha-1-\mathrm{a}$ antagonists and neuropeptide antagonists. Several protocols and different reaction condition have been employed to improve the yield of Biginelli reaction product [15-18]. These facts and usefulness of Biginelli reaction inspired us to synthesize such type of new compounds to investigate promising biological activities.

To the best of our knowledge, there are no reports on one step synthesis of pyrimidine derivatives using aromatic aldehydes, malononitrile and thiourea / urea. Therefore, we tried to synthesize 6-amino-5-cyano-4-phenyl-2-mercapto pyrimidine and its different hydroxyl derivatives in order to investigate new biological active compounds.

As part of our efforts to design and synthesize new pyrimidine derivatives [19], in this letter we would like to report the synthesis of 6-amino-5cyano 4-phenyl 2-mercapto pyrimidine and its hydroxyl derivative by three-component condensation of aromatic aldehydes, malononitrile and thiourea or urea using phosphorus pentoxide in absolute ethanol under refluxing condition.

\section{RESULTS AND DISCUSSION}

During the progress of reaction, the activated malononitrile is likely to be formed via a Knoevenagel condensation reaction of aromatic aldehydes and malononitrile. These further reacted with thiourea/urea to form desired product (Scheme 2).

In the presence of phosphorus pentoxide, reaction proceeds smoothly giving desired products in short time and in a quantitative yield. The formation of the product takes place when aryl aldehydes were reacted with malononitrile 


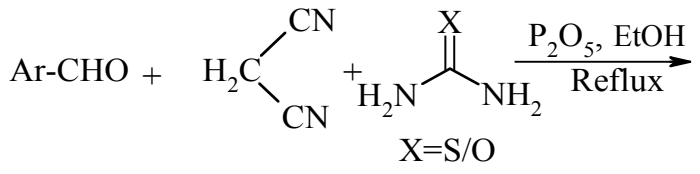

\}

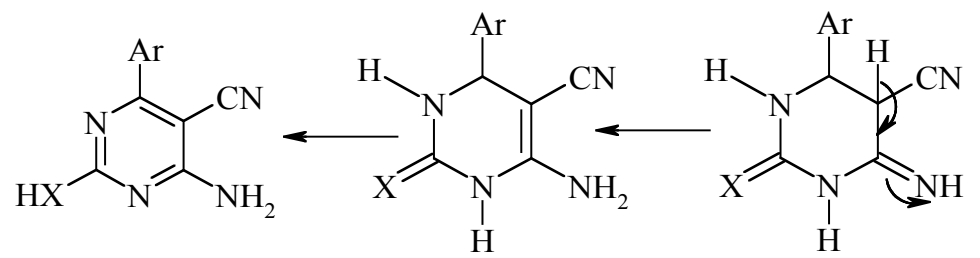<smiles>[X]C(N)=CCC(C)=C(C)C#N</smiles>

\section{Scheme 2.}

to form arylmethylene malononitrile, which subsequently reacted with thiourea or urea to form desired product.

Phosphorus pentoxide is inexpensive reagent and has tendency to absorb water molecules. The latter property of phosphorus pentoxide enhances the rate of reaction as onewater molecules are formed during the progress of the reaction. Therefore isolation of product was much easier. These results introduce another important application of phosphorus pentoxide in organic synthesis.

As a trial case, p-chlorobenzaldehyde (10mmol), malononitrile $(10 \mathrm{mmol})$ and phosphorus pentoxide were mixed thoroughly in $25 \mathrm{ml}$ absolute ethyl alcohol, the resulting reaction mixture was stirred mechanically for at least 10 minutes and then thiourea $(20 \mathrm{mmol})$ was added and the resulting reaction mixture was refluxed on water bath still completion of reaction. The reaction progress was monitored using TLC on silica gel $60 \mathrm{~F} 254$ plates. After the completion of reaction, the mixture was poured on crushed ice (about 200gm). The separated solid was filtered, dried and recrystalized from ethanol.

It was observed that the electron donating groups as well as electron with drawing groups present in aryl aldehydes do not affect the yield of the reaction. The physical and analytical data of synthesized compounds have been given in Table 1.

\section{EXPERIMENTAL}

The melting points were taken in open capillaries and were found to uncorrected. All the above products were characterized by proton NMR, IR and ${ }^{13} \mathrm{C}$ NMR The ${ }^{1} \mathrm{HNMR}$ and ${ }^{13} \mathrm{C}$ spectra were recorded by using $\mathrm{CDCl}_{3}+$ DMSO- $\mathrm{d}_{6}$ solvent on Brucker $400 \mathrm{MHz}$ spectrometer with tetra methyl silane as an internal standard. The reaction progress was monitored by TLC on Silica gel 60 F 254 plates.

\section{GENERAL PROCEDURE}

In a $250 \mathrm{ml}$ round bottom flask, aromatic aldehyde (10 mmol), malononitrile (10 $\mathrm{mmol})$ and phosphorus pentoxide 0.500 gms $(3.54 \mathrm{mmol})$ have stirred mechanically for ten
Table 1. The Physical and Analytical Data of Synthesized Compounds

\begin{tabular}{|c|c|c|c|c|}
\hline Sr. No. & Ar-CHO & $\mathbf{X}$ & Time (hrs) & Yield* \\
\hline \hline a. & $4-\mathrm{ClC}_{6} \mathrm{H}_{4-}$ & $\mathrm{S}$ & 1.0 & 92 \\
\hline b. & $4-\mathrm{N}, \mathrm{N}-(\mathrm{CH} 3)_{2} \mathrm{C}_{6} \mathrm{H}_{4^{-}}$ & $\mathrm{S}$ & 1.5 & 87 \\
\hline c. & $3-\mathrm{NO}_{2} \mathrm{C}_{6} \mathrm{H}_{4} 4^{-}$ & $\mathrm{S}$ & 1.5 & 89 \\
\hline d. & $3,4,\left(\mathrm{OCH}_{3}\right) \mathrm{C}_{6} \mathrm{H}_{3^{-}}$ & $\mathrm{O}$ & 2.0 & 89 \\
\hline e. & $\mathrm{C}_{6} \mathrm{H}_{5}-\mathrm{CH}=\mathrm{CH}-$ & $\mathrm{O}$ & 2.0 & 85 \\
\hline f. & $2-\mathrm{NO}_{2} \mathrm{C}_{6} \mathrm{H}_{4} 4^{-}$ & $\mathrm{O}$ & 1.5 & 80 \\
\hline g. & $\mathrm{C}_{6} \mathrm{H}_{5}{ }^{-}$ & $\mathrm{S}$ & 2.5 & 81 \\
\hline h. & $2-\mathrm{ClC}_{6} \mathrm{H}_{4}{ }^{-}$ & $\mathrm{O}$ & 2.0 & 83 \\
\hline i. & 3- $\mathrm{ClC}_{6} \mathrm{H}_{4^{-}}$ & $\mathrm{S}$ & 1.5 & 84 \\
\hline j. & 3- $\mathrm{ClC}_{6} \mathrm{H}_{4^{-}}$ & $\mathrm{O}$ & 1.5 & 88 \\
\hline
\end{tabular}

minutes in $25 \mathrm{ml}$ absolute ethanol and then thiourea/ urea (20mmol), added and mixed thoroughly. The resulting reaction mixture was heated at reflux using a water bath. The reaction mixture was poured on the crushed ice (about 200 gm) after the completion of the reaction monitored by TLC. On stirring separation of desired product takes place. The solid was filtered, washed with petroleum ether, dried and recrystalized by using ethanol.

\section{CONCLUSION}

In conclusion, we have developed a simple, quick and efficient method for the synthesis of 6-amino-5-cyano-4phenyl-2-mercapto pyrimidine and its hydroxyl derivative using phosphorus pentoxide. This new procedure is much more efficient, apart from its simplicity, the important advantage of the present procedure is the ability to tolerate variations in all the three components of the reaction. To the best of our knowledge, this is one of the quickest, economical and simple alternatives towards the synthesis of 6-amino-5-cyano-4-phenyl-2-mercapto pyrimidine. Ease of 
separation of pure product, selectively and in high yields in comparison to the two-step strategies, are a few of the unique features of this method.

\section{SPECTROSCOPIC DATA OF SYNTHESIZED PRO- DUCTS}

1) 6-Amino-5-Cyano-4-(4Chloro)-Phenyl-2-Mercapto Pyrimidine (Entry a)

m.p. $\left(135^{\circ} \mathrm{C}\right)$ IR (KBr): 3373, 3019, 2400, 2232, 1585, $1589,1406,1215,1096,828,780,617 \mathrm{~cm}^{-1} \mathrm{PMR}\left(\mathrm{CDCl}_{3}+\right.$ $\mathrm{DMSO}_{-} \mathrm{d}_{6} \delta 1.6(\mathrm{~s}, 1 \mathrm{H},-\mathrm{SH}), 7.51-7.53(\mathrm{dd}, 2 \mathrm{H}, \mathrm{Ar}-\mathrm{H})$, 7.84-7.87(dd, 2H, Ar-H), 3.9(br.s, 2H, $\left.-\mathrm{NH}_{2}\right){ }^{13} \mathrm{C} \mathrm{NMR}$ $\left(\mathrm{DMSO}-\mathrm{d}_{6}\right) \delta 92.3,117.9,121.7,129.3,130.1,131.8,136.5$, 158.2, 174.6.

2) 6-Amino-5-Cyano-4-(N-N Dimethyl Amino)-Phenyl-2Mercapto Pyrimidine. (Entry b)

m.p. $\left(172^{\circ} \mathrm{C}\right)$ IR $(\mathrm{KBr}): 3409,3019,2976,2221,1609$, $1565,1523,1381,1173,1046,819,669 \mathrm{~cm}^{-1} \mathrm{PMR}\left(\mathrm{CDCl}_{3}+\right.$ DMSO-d $\left.{ }_{6}\right) 1.58(\mathrm{~s}, 1 \mathrm{H},-\mathrm{SH}), 3.49\left(\mathrm{~s}, 6 \mathrm{H},-\mathrm{N}-\left(\mathrm{CH}_{3}\right)_{2}\right) 6.67-$ 6.70(dd, 2H, Ar-H), 7.84-7.85(dd, 2H, Ar-H), 7.46(br.s, 2H, $\left.-\mathrm{NH}_{2}\right){ }^{13} \mathrm{C}$ NMR (DMSO-d 6 ) $\delta 16.5,106.4,114.8,116.9$, 119.1, 133.8, 143.4, 147.9, 158.1, 166.9.

3) 6-Amino-5-Cyano-4-(3-Nitro)-Phenyl-2-Mercapto Pyrimidine (Entry c)

m.p. $\left(220^{\circ} \mathrm{C}\right) \mathrm{IR}(\mathrm{KBr}): 3361,3234,2926,2430,2208$, $1630,1529,1350,1100,808,696 \mathrm{~cm}^{-1} \mathrm{PMR}\left(\mathrm{CDCl}_{3}+\right.$ $\mathrm{DMSO}_{6}$ ) $\delta 2.5$ (s, 1H, -SH), 3.38 (br. s, 2H, -NH2), 7.537.73(m, 4H, Ar-H), ${ }^{13} \mathrm{C}$ NMR (DMSO-d 6 ) $\delta 107.7,120.3$, $132.3,134.2$, 135.8, 136.2, 138.3,140.7, 158.8, 159.7, 166.8.

\section{4) 6-Amino-5-Cyano-4-(3,4 dimethoxy)Phenyl-2-Hydroxy} Pyrimidine (Entry d)

m.p. $\left(167^{\circ} \mathrm{C}\right)$ IR (KBr): 3400, 3312, 3209, 2913, 2190, $1633,1545,1536,1347,1017,890,730 \mathrm{~cm}^{-1} \mathrm{PMR}\left(\mathrm{CDCl}_{3}+\right.$ DMSO-d $\left.{ }_{6}\right) \delta 3.94\left(\mathrm{~s}, 3 \mathrm{H},-\mathrm{OCH}_{3}\right), 3.99\left(\mathrm{~s}, 3 \mathrm{H},-\mathrm{OCH}_{3}\right)$, 6.95-6.97(dd, 1H, Ar-H), 7.37-7.39 (dd, 1H, Ar-H) 7.65(s, $1 \mathrm{H},-\mathrm{OH}), 7.68-7.60(\mathrm{dd}, 1 \mathrm{H}, \mathrm{Ar}-\mathrm{H}), 7.26$ (br.s, $\left.2 \mathrm{H},-\mathrm{NH}_{2}\right){ }^{13} \mathrm{C}$ NMR (DMSO-d $)_{6} \delta 23.4$ 25.3, 111.2, 118.4, 122.8, 126.9 $128.5,129.3130 .6,133.2,136.7,142.4,168.2$.

\section{5) 6-Amino-5-Cyano-4-Cinnamyl-2-Hydroxy Pyrimidine (Entry e)}

m.p. $\left(190^{\circ} \mathrm{C}\right)$ IR $(\mathrm{KBr}): 3410,3233,3125,3086,2929$, $2225,1687,1526,1452,1307,1071,756 \mathrm{~cm}^{-1} \mathrm{PMR}\left(\mathrm{CDCl}_{3}\right.$ + DMSO-d $\left._{6}\right) \delta 2.5$ (br.s, $\left.2 \mathrm{H},-\mathrm{NH}_{2}\right), 3.34(\mathrm{~s}, 1 \mathrm{H},-\mathrm{OH})$, 4.52(d, $1 \mathrm{H}, \mathrm{Ar}-\mathrm{CH}=\mathrm{CH}), 6.63(\mathrm{~d}, 1 \mathrm{H}, \mathrm{Ar}-\mathrm{CH}=\mathrm{CH}), 7.32(\mathrm{~m}$, $5 \mathrm{H}, \mathrm{Ar}-\mathrm{H}){ }^{13} \mathrm{C}$ NMR (DMSO-d 6 ) $\delta 78,106.3,126.1,126.4$, 127.3, 128.4, 133.4, 136.6 142.6, 154.9, 164.2.

6) 6-Amino-5-Cyano-4-(2-Nitro)-Phenyl-2-Hydroxy Pyrimidine (Entry f)

m.p. $\left(199^{\circ} \mathrm{C}\right)$ IR $(\mathrm{KBr}): 3341,3264,2910,2430,2200$, $1643,1529,1347,1123,848,687 \mathrm{~cm}^{-1} \mathrm{PMR}\left(\mathrm{CDCl}_{3}+\right.$ DMSO-d $\left.{ }_{6}\right) \delta 3.33$ (br.s, $2 \mathrm{H},-\mathrm{NH}_{2}$ ), 7.88-7.99(m, 2H, Ar-H), $8.31-8.33$ (dd, 2H, Ar-H) 8.06(s, 1H, -OH), ${ }^{13} \mathrm{C}$ NMR $\left(\right.$ DMSO $\left.\mathrm{d}_{6}\right) \delta 87.0,111.7,113.0,125.3,127.1,130.4,132.4$, $133.3,135.0,146.8,161.2$.
7) 6-Amino-5-Cyano-4-Phenyl-2-Mercapto Pyrimidine (Entry g)

m.p. $\left(175^{\circ} \mathrm{C}\right)$ IR (KBr):3464, 3322, 3219, 2927, 2227, 1623, 1586, 1522, 1347, 1017, $730 \mathrm{~cm}^{-} \mathrm{PMR}\left(\mathrm{CDCl}_{3}+\right.$ DMSO-d $\left.{ }_{6}\right) \delta 1.6(\mathrm{~s}, 1 \mathrm{H}, \mathrm{SH}), 3.6\left(\right.$ br.s, $\left.2 \mathrm{H}, \mathrm{NH}_{2}\right), 6.68-7.2$ $(\mathrm{m}, 5 \mathrm{H}, \mathrm{Ar}-\mathrm{H}),{ }^{13} \mathrm{C}$ NMR (DMSO-d 6 ) $\delta 107.2,118.5,122.6$, 126.4, 128.7, 132.3, 133.4, 136.7, 142.3, 166.2.

8) 6-Amino-5-Cyano-4-(2-Chloro)-Phenyl-2-Hydroxy Pyrimidine (Entry h)

m.p.( $\left.190^{\circ} \mathrm{C}\right)$ IR (KBr): 3463, 3325, 3321, 2977, 2226, $1625,1578,1440,1190,1018,886,785,749 \mathrm{~cm}^{-1}$ PMR $\left(\mathrm{CDCl}_{3}+\mathrm{DMSO}_{-} \mathrm{d}_{6}\right) \delta 3.4$ (br. s, $\left.1 \mathrm{H}, \mathrm{OH}\right), 7.50-7.7 .7(\mathrm{~m}$, $2 \mathrm{H}, \mathrm{Ar}-\mathrm{H}), 8.14-8.28(\mathrm{~m}, 2 \mathrm{H}, \mathrm{Ar}-\mathrm{H}), 2.5$ (br.s, $\left.2 \mathrm{H}, \mathrm{NH}_{2}\right),{ }^{13} \mathrm{C}$ NMR (DMSO-d $\left.{ }_{6}\right) \delta 81.8,126.5,128.8,131.6,134.8,139.7$, $141.9,152.9$

9) 6-Amino-5-Cyano-4-(3-Chloro)-Phenyl-2-Mercapto Pyrimidine (Entry i)

m.p. $\left(225^{\circ} \mathrm{C}\right) \mathrm{IR}(\mathrm{KBr}): 3315,3301,2935,2443,2222$, 1644, 1426, 1170, 1048, 876, 767 $\mathrm{cm}^{-1} \mathrm{PMR}(\mathrm{CDCl} 3+$ DMSO-d 6$) 1.5(\mathrm{~s}, 1 \mathrm{H},-\mathrm{SH}), 7.25(\mathrm{~s}, 1 \mathrm{H}, \mathrm{Ar}-\mathrm{H}), 7.50-7.65(\mathrm{~m}$ $1 \mathrm{H}, \mathrm{Ar}-\mathrm{H}), 7.67 .65(\mathrm{~d}, 1 \mathrm{H}, \mathrm{Ar}-\mathrm{H}), 7.85$ (br.s, $\left.2 \mathrm{H},-\mathrm{NH}_{2}\right),{ }^{13} \mathrm{C}$ NMR $\left(\right.$ DMSO$\left.^{-d_{6}}\right) \delta 110.2,126.2,128.2,130.4,130.9,132.4$, $134.3,158.0,166.8$.

10) 6-Amino-5-Cyano-4-(3-Chloro)-Phenyl-2-Hydroxy Pyrimidine (Entry j)

m.p. $\left(225^{\circ} \mathrm{C}\right) \mathrm{IR}(\mathrm{KBr}): 3445,3314,3018,2910,2220,1645$, $1578,1420,1140,1018,895,759 \mathrm{~cm}^{-1} \mathrm{PMR}(\mathrm{CDCl} 3+$ DMSO-d $\left.{ }_{6}\right) \delta 1.53\left(\mathrm{~s}, 2 \mathrm{H},-\mathrm{NH}_{2}\right), 7.26(\mathrm{~s}, 1 \mathrm{H}, \mathrm{Ar}-\mathrm{H}), 7.47-$ $7.62(\mathrm{~m}, 1 \mathrm{H}, \mathrm{Ar}-\mathrm{H}), 7.72(\mathrm{~s}, 1 \mathrm{H},-\mathrm{OH}), 7.82-7.85$ (m, 2H, Ar$\mathrm{H}){ }^{13} \mathrm{C}$ NMR $\left(\mathrm{DMSO}_{-} \mathrm{d}_{6}\right) \delta 102.6,121.4,125.4,128.3$, $130.9,131.0,134.3,138.2,140.1,158.1,171.7$.

\section{ACKNOWLEDGEMENT}

This work is supported by the University Grant Commission (UGC), New Delhi for providing grant to the Department of Chemistry Shivaji University under SAP programme. One of the authors (DRP) acknowledges receiving SAP fellowship under the scheme Research Fellowship in Sciences for Meritorious Students from UGC, New Delhi.

\section{REFERENCES}

[1] Brown, R.C. D. Recent developments in solid phase organic synthesis. J. Chem. Soc. Perkin Trans 1, 1998, 3293-320.

[2] Pershin, N. G.; Sherbakova, L. I.; Zykova, T. N.; Sakolova, V. N. Antibacterial activity of pyrimidine and pyrrolo-(3,2-d)-pyrimidine derivatives. Pharmacol. Taksiko., 1972, 35, 466-71

[3] Metolcsy, G. Structure-activity correlations and mode of action of some selected types of antifungal compounds. World Rev. Pest Contr. 1971, 10, 50-59; [Chem. Abstr. 1972, 76, 82031s].

[4] Regnier, G. L.; Canevar, R. J.; Canevar, J. C.; Douarec, L.; Halstop, S.; Daussy, J. Triphenylpropylpiperazine derivatives as new potent analgetic substances. J. Med. Chem., 1972, 15, 295-301

[5] Winter, C. A.; Fisley, E. A.; Nuss, G. W. Carrageenin-induced edema in hind paw of the rat as an assay for antiinflammatory drugs. Proc. Soc. Exp. Biol. Med., 1962, 111, 544-47.

[6] Suguira, A. F.; Schmid, M.M.; Brown, F.G. Effect of compounds on a spectrum of rat tumors. Cancer Chemother. Rep. Part 2, 1973, $3(1), 231-38$. 
[7] Mojtahedi, M. M.; Saidi, M. R.; Shirzi, J. S.; Bolourtchian, M. Microwave promoted efficient synthesis of substituted uracils and thiouracils under solvent-free conditions. Synth. Commun., 2002, $32,851-55$

[8] Ma, Y.; Qian, C.; Wang, L.; Yang, M. Lanthanide Triflate catalyzed biginelli reaction. One-pot synthesis of dihydropyrimidinones under solvent free conditions. Org. Chem., 2000, 65, 3864-68

[9] Kambe, S.; Salto, K. H.; A one pot synthesis of 4-oxo-2thioxopyrimidine derivatives by the ternary condensation of ethyl cyanoacetate, aldehydes, and thiourea. Synthesis, 1979, 4, 287-89.

[10] Ji-Tai, Li.; Zhi-Ping Han, Lin.; Jun-Fen Li.; Tong-Shuang, One-pot synthesis of 4-oxo-2-thioxohexahydropyrimidines catalyzed by potassium carbonate under ultrasound. Synth. Commun., 2004, 34, 2623-31.

[11] Russell, Hitchings, H.G. Some 2,4,6-triamino-S-alkyl and SBenzylpyrimidines. J. Am. Chem. Soc., 1952, 74, 3443-44.

[12] Biginelli, P. Aldehyde-urea derivatives of aceto- and oxaloacetic acids. Gazz. Chim. Ital., 1893, 23, 360-413.

[13] Schnell, B.; Krenn, W.; Faber, K.; Kappe, C. O. Synthesis and reactions of Biginelli-compounds. Part23. Chemoenzymatic syntheses of enantiomerically pure 4-aryl-3,4-dihydropyrimidin2(1H)-ons. J. Chem. Soc. Perkin Trans 1., 2000, 4382-89.

[14] Kappe, C. O. 100 years of the biginelli dihydropyrimidine synthesis. Tetrahedron, 1993, 49, 6937-63.
[15] Kappe, C. O. Recent Advances in the Biginelli Dihydropyrimidine Synthesis. New Tricks from an Old Dog. Acc. Chem. Res., 2000, 33, 879-88.

[16] Srinivas, K. V. N. S.; Das, B. iodine catalyzed one-pot synthesis of 3,4-dihydropyrimidin-2 $(1 H)$-ones and thiones: a simple and efficient procedure for the biginelli reaction. Synthesis, 2004, 13, 2091-93.

[17] Sun, Q.; Ge. Y.; Wang Z.; Li, T.; Chang, R. A highly efficient solvent-free Synthesis of dihydropyrimidinones catalyzed by zinc chloride. Synthesis, 2004, 1047-51.

[18] Wang, Z.; Xu, L.; Wang, H. Novel biginelli-like three-component cyclocondensation reaction: efficient synthesis of 5-unsubstituted 3,4-dihydropyrimidin-2(1H)-ones. Tetrahedron Lett., 2004, 45, 7951-53.

[19] (a) Deshmukh, M.B.; Salunkhe, S.M.; Patil, D. R.; Anbhule, P.V. A novel and efficient one step synthesis of 2-amino-5-cyano-6hydroxy-4-arylpyrimidines and their anti-bacterial activity Eur. $J$. Med. Chem. 2009, 44, 2651-54. (b) Deshmukh, M.B.; Anbhule, P.V.; Jadhav, S.D.; Mali, A. R.; Jagtap, S. S.; Deshmukh, S. A. An efficient, simple, one pot synthesis of dihydropyrymidine- $2(1 \mathrm{H})$ one using phosphorus pentoxide. Indian J. Chem. 2007, 42B, 144548. (c) Deshmukh M.B.; Anbhule, P.V.; Jadhav, S.D.; Jagtap, S.S.; Patil, D.R.; Salunkhe, S.M.; Sankpal, S.A. A novel and environmental friendly one-step synthesis of 2,6-diamino-4phenylpyrimidine-5-carbonitrile using potassium carbonate in water. Indian J. Chem., 2008, 47B, 792-95. 